\title{
Applications of General Residual Power Series Method to Differential Equations with Variable Coefficients
}

\author{
Bochao Chen, ${ }^{1}$ Li Qin, ${ }^{1}$ Fei Xu, ${ }^{1,2}$ and Jian $\mathrm{Zu} \mathbb{D}^{1,3}$ \\ ${ }^{1}$ School of Mathematics and Statistics and Center for Mathematics and Interdisciplinary Sciences, Northeast Normal University, \\ Changchun 130024, China \\ ${ }^{2}$ College of Mathematics, Jilin University, Changchun 130012, China \\ ${ }^{3}$ Key Laboratory of Symbolic Computation and Knowledge Engineering of Ministry of Education, Jilin University, \\ Changchun 130012, China \\ Correspondence should be addressed to Jian Zu; zuj100@nenu.edu.cn
}

Received 21 March 2018; Revised 24 May 2018; Accepted 11 June 2018; Published 9 July 2018

Academic Editor: Jorge E. Macias-Diaz

Copyright (C) 2018 Bochao Chen et al. This is an open access article distributed under the Creative Commons Attribution License, which permits unrestricted use, distribution, and reproduction in any medium, provided the original work is properly cited.

\begin{abstract}
This paper is devoted to studying the analytical series solutions for the differential equations with variable coefficients. By a general residual power series method, we construct the approximate analytical series solutions for differential equations with variable coefficients, including nonhomogeneous parabolic equations, fractional heat equations in $2 \mathrm{D}$, and fractional wave equations in 3D. These applications show that residual power series method is a simple, effective, and powerful method for seeking analytical series solutions of differential equations (especially for fractional differential equations) with variable coefficients.
\end{abstract}

\section{Introduction}

In the field of science and engineering, many physical phenomena can be described by differential equations with variable coefficients. For example, some physical problems in inhomogeneous media [1-3]. In the past, many assumptions on integral order differential equations were applied artificially to describe the systems with memory properties and hereditary properties. Some significant information will be lost by such assumptions. Generally, fractional calculus provides an effective tool to describe memory properties and hereditary properties of different materials and processes without extra assumptions. Now the fractional differential equation has attracted a great deal of interest in several areas including chemistry, physics, engineering, and even finance and social sciences $[4,5]$. Some recent progress in fractional calculus can be found in [6-8].

The analytical series solutions of differential equations are of fundamental importance in applied science. Various numerical and analytical methods are proposed such as Adomian decomposition method $[9,10]$, Fractional complex transform method [11], and Laplace transform method [12].
Although lots of methods are put forward, scientists are still looking for more effective ways to solve specific problems, especially for the fractional equations with variable coefficients.

The residual power series method (RPS), proposed by Abu Arqud in [13], is an efficient and easy method for constructing power series solutions of differential equations without linearization, perturbation, or discretization. Different from the classical power series method, RPS does not need to compare the coefficients of the corresponding terms. This method computes the coefficients of the power series by a chain of equations with one or more variables. One advantage is that RPS is not affected by computational round-off errors and also does not require large computer memory and extensive time. In [14], power series solutions of higher-order ordinary differential equations are obtained by RPS. Inspired by this approach, we present a general residual power series method (GRPS) for constructing power series solutions of time-space fractional differential equations with variable coefficients:

$$
D_{t}^{m \alpha} u(\boldsymbol{x}, t)+P(\boldsymbol{x}) G(u)=F(\boldsymbol{x}, t),
$$




$$
\begin{aligned}
D_{t}^{i \alpha} u(\boldsymbol{x}, 0)=a_{i}(\boldsymbol{x}), & \\
& i=0, \ldots, m-1,
\end{aligned}
$$

where $\boldsymbol{x}=\left(x_{1}, x_{2}, \ldots, x_{d}\right) \in \mathbb{R}^{d}, \alpha \in((m-1) / m, 1], m \in \mathbb{N}^{+}$, and

$$
\begin{aligned}
& G(u):=G\left(u, D_{t}^{\alpha} u, \ldots, D_{t}^{(m-1) \alpha} u, D_{x_{1}}^{\beta_{11}} u, \ldots, D_{x_{d}}^{\beta_{l d}} u, \ldots,\right. \\
& \left.\quad D_{x_{1}}^{\beta_{l 1}} u, \ldots, D_{x_{d}}^{\beta_{l d}} u\right)
\end{aligned}
$$

with $p-1<\beta_{p j} \leqslant p, p=1, \ldots, l ; j=1, \ldots, d$. Here $D_{t}^{i \alpha}$ and $D_{x_{j}}^{\beta_{p j}}$ mean the Caputo fractional derivative with respect to $t$ of order $i \alpha$ and $x_{j}$ of order $\beta_{p j}$, respectively. Such type of differential equation provides an exact description of some physical phenomena in fluid dynamics, electrodynamics, and elastic mechanics.

RPS has been extended to many partial differential equations (PDE), especially to fractional partial differential equations (FPDE), such as time-fractional dispersive PDE $[15,16]$, time-fractional KdV-Burgers equations [17], homogeneous time-fractional wave equation [18], and time-space fractional Boussinesq equations [19]. In the present paper, we will apply GRPS to a series of PDE with variable coefficients, including fourth-order parabolic equations, fractional heat equation, and fractional wave equation. For other approximation and numerical techniques for FPDE, we refer to finite difference methods [20,21], differential transform method $[22,23]$, wavelet method [24], Adomian's decomposition method [25], variational iteration method [26, 27], homotopy analysis method [28], homotopy perturbation method [29], tau method [30, 31], and so on.

The paper is organized as follows: some necessary definitions and theorems will be presented in Section 2. In Section 3, we propose the main steps of GRPS for the general time-space fractional equations with variable coefficients. In Section 4, the applications of GRPS to some different equations with variable coefficients are given, including fourthorder parabolic equations, fractional heat equations, and fractional wave equations. Finally, conclusions are presented in Section 5.

\section{Concepts on Fractional Calculus Theory}

There are several definitions of the fractional integration with order $\alpha \geq 0$, and they are not necessarily equivalent to each other. The two most common ones are Riemann-Liouville's definition and Caputo's definition; see $[32,33]$.

Definition 1. The Mittag-Leffler function is defined as follows:

$$
E_{\alpha}(x):=\sum_{k=0}^{\infty} \frac{x^{k}}{\Gamma(\alpha k+1)}, \quad \alpha>0
$$

Definition 2. A real function $u(x, t), x \in I, t>0$ is said to be in the space $C_{\mu}\left(I \times \mathbb{R}^{+}\right), \mu \in \mathbb{R}$, if there exists a real number $p>\mu$ such that $u(x, t)=t^{p} f(x, t)$, where $f(x, t) \in C\left(I \times \mathbb{R}^{+}\right)$, and it is said to be in the space $C_{\mu}^{n}$, if $\left(\partial^{n} / \partial t^{n}\right) u(x, t) \in C_{\mu}$, $n \in \mathbb{N}$.

Definition 3. Let $u(x, t) \in C_{\mu}\left(I \times \mathbb{R}^{+}\right), \mu \geq-1$. The RiemannLiouville fractional integral operator of order $\alpha \geq 0$ of $u(x, t)$ is defined as follows:

$$
\begin{aligned}
& J_{t}^{\alpha} u(x, t) \\
& := \begin{cases}\frac{1}{\Gamma(\alpha)} \int_{0}^{t}(t-\tau)^{\alpha-1} u(x, \tau) \mathrm{d} \tau, & \alpha>0, x \in I, t>\tau \geq 0, \\
u(x, t), & \alpha=0 .\end{cases}
\end{aligned}
$$

Definition 4. The Caputo time-fractional derivative operator of order $\alpha$ of $u(x, t)$ is defined as follows:

$$
\begin{aligned}
& D_{t}^{\alpha} u(x, t) \\
& \quad:= \begin{cases}J_{t}^{n-\alpha}\left(\frac{\partial^{n} u(x, t)}{\partial t^{n}}\right), & n-1<\alpha<n, n \in \mathbb{N}^{+}, \\
\frac{\partial^{n} u(x, t)}{\partial t^{n}}, & \alpha=n .\end{cases}
\end{aligned}
$$

Definition 5. The Caputo space fractional derivative operator of order $\beta$ is defined as follows:

$$
\begin{aligned}
& D_{x}^{\beta} u(x, t) \\
& := \begin{cases}J_{x}^{n-\beta}\left(\frac{\partial^{n} u(x, t)}{\partial x^{n}}\right), & n-1<\beta<n, n \in \mathbb{N}^{+}, \\
\frac{\partial^{n} u(x, t)}{\partial x^{n}}, & \alpha=n .\end{cases}
\end{aligned}
$$

Definition 6. A power series representation of the form

$$
\sum_{n=0}^{\infty} c_{n}\left(t-t_{0}\right)^{n \alpha}:=c_{0}+c_{1}\left(t-t_{0}\right)^{\alpha}+c_{2}\left(t-t_{0}\right)^{2 \alpha}+\cdots
$$

is called a fractional power series (FPS) about $t_{0}$, where $t$ is a variable and $c_{n}$ are the coefficients of the series.

Theorem 7 (see [34]). Suppose that $f$ has a FPS representation at $t_{0}$ of the form

$$
f(t)=\sum_{n=0}^{\infty} c_{n}\left(t-t_{0}\right)^{n \alpha}, \quad t_{0} \leqslant t<t_{0}+R
$$

where $R$ is the radius of convergence of the FPS. If $D_{t}^{n \alpha} f(t) \epsilon$ $C\left(t_{0}, t_{0}+R\right)$ for $n=0,1,2, \cdots$, then the coefficients $c_{n}$ will take the form of

$$
c_{n}=\frac{\left.D_{t}^{n \alpha} f(t)\right|_{t=t_{0}}}{\Gamma(n \alpha+1)}
$$

where $D_{t}^{n \alpha}=D_{t}^{\alpha} \cdot D_{t}^{\alpha} \cdots D_{t}^{\alpha}$ (n-times).

\section{Algorithm of GRPS}

In this section, we give a general RPS to obtain fractional power series solutions for any-order time-space fractional 
differential equations with variable coefficients (1). The analytic function $u(\boldsymbol{x}, t)$ can be expanded as follows:

$$
\begin{aligned}
u(\boldsymbol{x}, t)=\sum_{n=0}^{\infty} u_{n}(\boldsymbol{x}, t):=\sum_{n=0}^{\infty} C_{n}(\boldsymbol{x}) t^{n \alpha}, & \\
x & \in I \subset \mathbb{R}^{d},|t|<R,
\end{aligned}
$$

where $R$ is the radius of convergence of above series. Substitute the initial conditions in (1); we have

$$
a_{i}(\boldsymbol{x})=C_{i}(\boldsymbol{x}) \Gamma(i \alpha+1), \quad i=0,1, \ldots, m-1,
$$

which implies

$$
\begin{aligned}
u_{i}(\boldsymbol{x}, t)=C_{i}(\boldsymbol{x}) t^{i \alpha}=\frac{a_{i}(\boldsymbol{x})}{\Gamma(i \alpha+1)} t^{i \alpha}, & \\
& i=0,1, \ldots, m-1 .
\end{aligned}
$$

So we have the initial guess approximation of $u(x, t)$ in the following form:

$$
\begin{aligned}
u^{\text {initial }}(\boldsymbol{x}, t):= & u_{0}(\boldsymbol{x}, t)+u_{1}(\boldsymbol{x}, t)+\cdots+u_{m-1}(\boldsymbol{x}, t) \\
= & a_{0}(\boldsymbol{x})+\frac{a_{1}(\boldsymbol{x})}{\Gamma(\alpha+1)} t^{\alpha}+\cdots \\
& +\frac{a_{m-1}(\boldsymbol{x})}{\Gamma((m-1) \alpha+1)} t^{(m-1) \alpha} .
\end{aligned}
$$

Define the approximate solution of (1) by the $k$ th truncated series:

$$
\begin{aligned}
u^{k}(\boldsymbol{x}, t):=u^{\text {initial }}(\boldsymbol{x}, t)+\sum_{i=m}^{k} C_{i}(\boldsymbol{x}) t^{i \alpha}, \\
\quad \\
\quad k=m, m+1, m+2 \cdots .
\end{aligned}
$$

Before applying GRPS to solve (1), we give some notations

$$
\operatorname{Res}(u, \boldsymbol{x}, t):=D_{t}^{m \alpha} u(\boldsymbol{x}, t)+P(\boldsymbol{x}) G(u)-F(\boldsymbol{x}, t) .
$$

Substituting the $k$ th truncated approximate solutions $u^{k}(x, t)$ into (1), we obtain the $k$ th residual function

$$
\begin{aligned}
\operatorname{Res}^{k}(u, \boldsymbol{x}, t):= & D_{t}^{m \alpha} u^{k}(\boldsymbol{x}, t)+P(\boldsymbol{x}) G^{k}(u) \\
& -F(\boldsymbol{x}, t),
\end{aligned}
$$

where

$$
\begin{aligned}
& G^{k}(u)=G\left(u^{k}, D_{t}^{\alpha} u^{k}, \ldots, D_{t}^{(m-1) \alpha} u^{k}, D_{x_{1}}^{\beta_{11}} u^{k}, \ldots, D_{x_{d}}^{\beta_{1 d}} u^{k},\right. \\
& \left.\ldots, D_{x_{1}}^{\beta_{l 1}} u^{k}, \ldots, D_{x_{d}}^{\beta_{l d}} u^{k}\right) .
\end{aligned}
$$

Then we have the following facts:

(1) $\lim _{k \rightarrow \infty} u^{k}(\boldsymbol{x}, t)=u(\boldsymbol{x}, t)$;

(2) $\operatorname{Res}(u, x, t)=0$;

(3) $\lim _{k \rightarrow \infty} \operatorname{Res}^{k}(u, \boldsymbol{x}, t)=\operatorname{Res}(u, \boldsymbol{x}, t), \boldsymbol{x} \in I \subseteq \mathbb{R}^{d},|t|$ $<R$.
Assume that

$$
\left.D_{t}^{(k-m) \alpha} \operatorname{Res}^{k}(u, x, t)\right|_{t=0}=0 .
$$

Since

$$
\begin{aligned}
\left.D_{t}^{(k-m) \alpha} \operatorname{Res}^{k}(u, \boldsymbol{x}, t)\right|_{t=0} \\
=C_{k}(\boldsymbol{x}) \Gamma(k \alpha+1) \\
\quad+D_{t}^{(k-m) \alpha}\left[P(\boldsymbol{x}) G^{k}(u)-F(\boldsymbol{x}, t)\right]_{t=0},
\end{aligned}
$$

we have

$$
\begin{gathered}
C_{k}(\boldsymbol{x})=-\frac{D_{t}^{(k-m) \alpha}\left[P(\boldsymbol{x}) G^{k}(u)-F(\boldsymbol{x}, t)\right]_{t=0}}{\Gamma(k \alpha+1)}, \\
k=m, m+1, m+2, \cdots .
\end{gathered}
$$

In fact, this relation is a fundamental rule in GRPS. So the FPS solution of (1) is

$$
\begin{aligned}
u(\boldsymbol{x}, t) & =u^{\text {initial }}(\boldsymbol{x}, t)+\sum_{i=m}^{\infty} C_{i}(\boldsymbol{x}) t^{i \alpha} \\
& =\sum_{i=0}^{m-1} \frac{a_{i}(\boldsymbol{x})}{\Gamma(i \alpha+1)} t^{i \alpha}+\sum_{i=m}^{\infty} \frac{f_{i}(\boldsymbol{x})}{\Gamma(i \alpha+1)} t^{i \alpha},
\end{aligned}
$$

where

$$
\begin{aligned}
& f_{k}(\boldsymbol{x})=-D_{t}^{(k-m) \alpha}\left[P(\boldsymbol{x}) G^{k}(u)-F(\boldsymbol{x}, t)\right]_{t=0}, \\
& k=m, m+1, m+2, \cdots .
\end{aligned}
$$

\section{Applications of GRPS to PDEs with Variable Coefficients}

4.1. Fourth-Order Parabolic Equation with Variable Coefficients in $\mathbb{R}^{1}$. Let us consider the fourth-order parabolic differential equation

$$
\frac{\partial^{2} u}{\partial t^{2}}+\left(\frac{x}{\sin x}-1\right) \frac{\partial^{4} u}{\partial x^{4}}=0, \quad 0<x<1, t>0
$$

where $x / \sin x-1>0$ is the ratio of flexural rigidity of the beam to its mass per unit length; see [35]. In [35], the initial conditions and the boundary conditions of (23) are

$$
\begin{gathered}
u(x, 0)=x-\sin x, \\
\frac{\partial}{\partial t} u(x, 0)=-x+\sin x,
\end{gathered}
$$

and

$$
\begin{aligned}
u(0, t) & =0, \\
u(1, t) & =e^{-t}(1-\sin 1), \\
\frac{\partial^{2}}{\partial x^{2}} u(0, t) & =0, \\
\frac{\partial^{2}}{\partial x^{2}} u(1, t) & =e^{-t} \sin 1,
\end{aligned}
$$


respectively. According to (10), $u$ can be written in the following form:

$$
\begin{aligned}
u(x, t)= & C_{0}(x)+C_{1}(x) t+C_{2}(x) t^{2}+C_{3}(x) t^{3} \\
& +\cdots
\end{aligned}
$$

The initial approximation is

$$
u^{\text {initial }}(x, t)=(x-\sin x)-(x-\sin x) t .
$$

Now by (15), denote

$$
\begin{aligned}
\operatorname{Res}(x, t) & =\frac{\partial^{2} u(x, t)}{\partial t^{2}}+\left(\frac{x}{\sin x}-1\right) \frac{\partial^{4} u(x, t)}{\partial x^{4}}, \\
u^{k}(x, t) & =u^{\text {initial }}+\sum_{i=2}^{k} f_{i}(x) t^{i}, \\
\operatorname{Res}^{k}(x, t) & =\frac{\partial^{2} u^{k}(x, t)}{\partial t^{2}}+\left(\frac{x}{\sin x}-1\right) \frac{\partial^{4} u^{k}(x, t)}{\partial x^{4}} .
\end{aligned}
$$

By (16) and (18), we assume that

$$
D_{t}^{k-2} \operatorname{Res}^{k}(x, 0)=0, \quad k=2,3,4 \cdots .
$$

Letting $k=2$ in (29), it shows

$$
2 ! C_{2}(x)+\left(\frac{x}{\sin x}-1\right)(-\sin x)=0,
$$

which implies

$$
C_{2}(x)=\frac{x-\sin x}{2 !}
$$

So the 2 nd truncated approximate solution of (23) is

$$
u^{2}(x, t)=(x-\sin x)-(x-\sin x) t+\frac{x-\sin x}{2 !} t^{2} .
$$

Similarly, $C_{k}(x)$ can be constructed as follows:

$$
C_{k}(x)=\frac{(-1)^{k}}{k !}(x-\sin x), \quad k=2,3,4 \cdots .
$$

So the $k$ th truncated approximate solution of (23) is

$$
u^{k}(x, t)=(x-\sin x) \sum_{i=0}^{k} \frac{(-1)^{k}}{k !} t^{i}
$$

Finally, if we define

$$
u(x, t):=\lim _{k \rightarrow \infty} u^{k}(x, t)=(x-\sin x) e^{-t},
$$

it is easy to verify that $u(x, t)$ in (35) is the exact solution of (23) with boundary value condition (25).

Numerical comparisons are studied next. Figure 1 shows the exact solution $u$ of (23) with $0 \leq t \leq 5$. In Figure $2, u^{9}, u^{10}$, $u^{11}$, and $u^{12}$ represent the 9th-, 10th-, 11th-, and 12th-order truncated approximate solution of $u$ with $0 \leq t \leq 5$. It shows that these GRPS approximate solutions are convergent to the exact solution $u$.

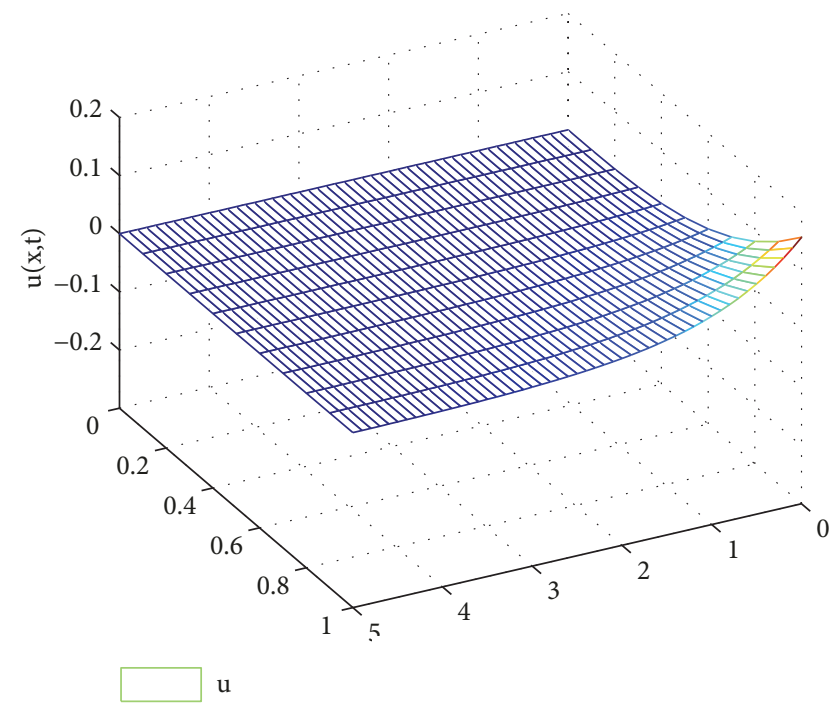

Figure 1: The exact solution $u$ of (23).
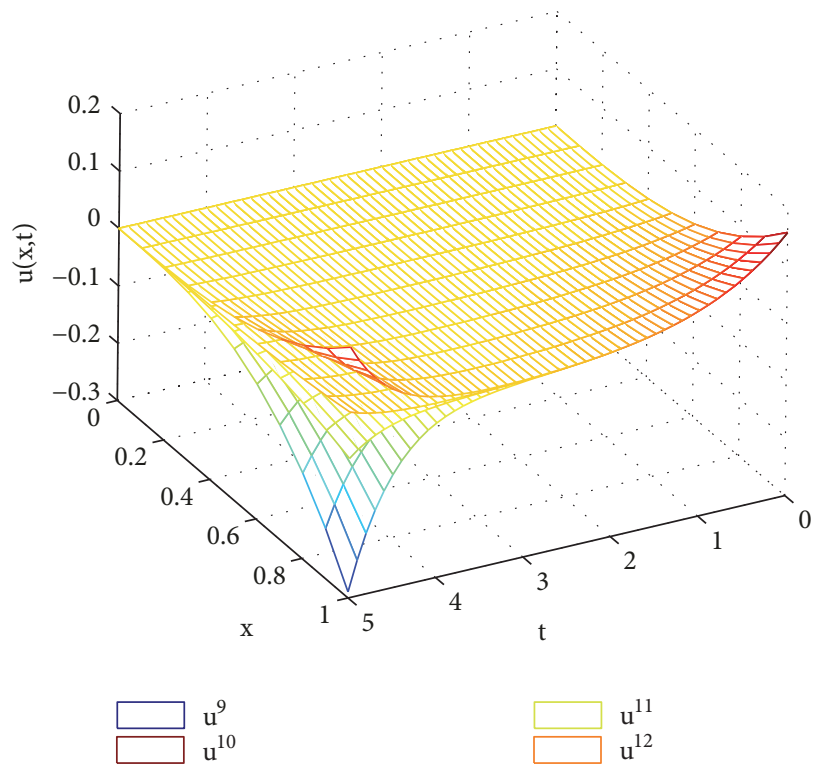

FIGURE 2: The approximate solutions $u^{9}, u^{10}, u^{11}$, and $u^{12}$ of (23).

4.2. Nonhomogeneous Parabolic Equation with Source Term in $\mathbb{R}^{1}$. Let us consider the nonhomogeneous parabolic equation (see [35]):

$$
\begin{aligned}
\frac{\partial^{2} u}{\partial t^{2}}+(1+x) \frac{\partial^{4} u}{\partial x^{4}}=\left(x^{4}+x^{3}-\frac{6}{7 !} x^{7}\right) \cos t & \\
0 & <x<1, t>0
\end{aligned}
$$

with the initial conditions

$$
\begin{aligned}
u(x, 0) & =\frac{6}{7 !} x^{7}, \\
\frac{\partial}{\partial t} u(x, 0) & =0,
\end{aligned}
$$


and the boundary conditions

$$
\begin{aligned}
u(0, t) & =0, \\
u(1, t) & =\frac{6}{7 !} \cos t, \\
\frac{\partial^{2}}{\partial x^{2}} u(0, t) & =0, \\
\frac{\partial^{2}}{\partial x^{2}} u(1, t) & =\frac{1}{20} \cos t .
\end{aligned}
$$

Assume that $u(x, t)$ is an analytical function with $t>0$. The initial approximation is

$$
u^{\text {initial }}(x, t)=\frac{6}{7 !} x^{7}
$$

Denote

$$
\begin{aligned}
\operatorname{Res}^{k}(x, t)= & \frac{\partial^{2} u^{k}(x, t)}{\partial t^{2}}+(1+x) \frac{\partial^{4} u^{k}(x, t)}{\partial x^{4}} \\
& -\left(x^{4}+x^{3}-\frac{6}{7 !} x^{7}\right) \cos t,
\end{aligned}
$$

where

$$
u^{k}(x, t)=u^{\text {initial }}(x, t)+C_{2}(x) t^{2}+\cdots+C_{k}(x) t^{k} .
$$

Let $k=2$ in (40); it yields

$$
\begin{aligned}
\operatorname{Res}^{2}(x, t)= & 2 C_{2}(x)+(1+x)\left(\frac{6}{3 !} x^{3}+C_{2}^{(4)}(x) t^{2}\right) \\
& -\left(x^{4}+x^{3}-\frac{6}{7 !} x^{7}\right) \cos t .
\end{aligned}
$$

Using the fact that

$$
D_{t}^{k-2} \operatorname{Res}^{k}(x, 0)=0, \quad k=2,3,4 \cdots,
$$

we have

$$
C_{2}(x)=-\frac{6}{2 ! 7 !} x^{7}
$$

Thus the 2nd truncated series have the following form:

$$
u^{2}(x, t)=\frac{6}{7 !} x^{7}-\frac{6}{2 ! 7 !} x^{7} t^{2}
$$

Similarly, taking $k=3,4,5,6$ in (40) we obtain

$$
\begin{aligned}
& C_{3}(x)=0, \\
& C_{4}(x)=\frac{6}{4 ! 7 !} x^{7}, \\
& C_{5}(x)=0, \\
& C_{6}(x)=-\frac{6}{6 ! 7 !} x^{7} .
\end{aligned}
$$

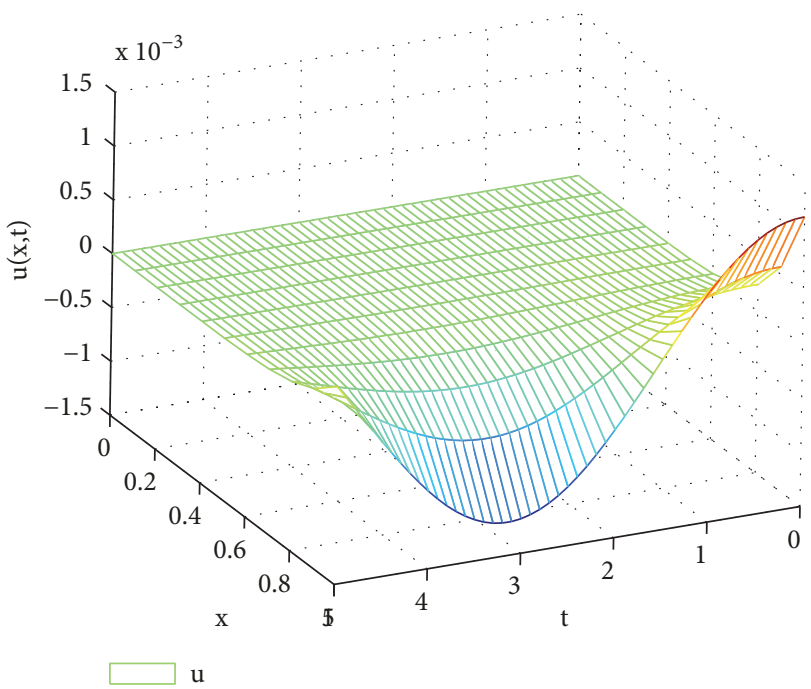

FIGURE 3: The exact solution $u$ of (36).

Then 6 th truncated approximate solution of (36) is

$$
u^{6}(x, t)=\frac{6}{7 !} x^{7}-\frac{6}{2 ! 7 !} x^{7} t^{2}+\frac{6}{4 ! 7 !} x^{7} t^{4}-\frac{6}{6 ! 7 !} x^{7} t^{6}
$$

By (21), we can obtain the solution of (36):

$$
u(x, t)=\lim _{k \rightarrow \infty} u^{k}(x, t)=\frac{6}{7 !} x^{7} \cos t,
$$

which is consistent with the solution obtained by Adomian decomposition method [35].

Some numerical comparisons are given next. Figure 3 shows the exact solution $u$ of (36) with $0 \leq t \leq 5$. In Figure 4, $u^{19}, u^{20}, u^{21}$, and $u^{22}$ represent the 19th-, 20th-, 21st-, and 22nd-order GRPS solution of $u$ with $0 \leq t \leq 5$. It shows that these approximate solutions are convergent to the exact solution $u$.

4.3. Fractional Heat Equation with Variable Coefficients in $\mathbb{R}^{2}$. Consider the two-dimensional heat equation with variable coefficients

$$
\begin{array}{r}
D_{t}^{\alpha} u(x, y, t)=\frac{1}{2} y^{2} u_{x x}(x, y, t)+\frac{1}{2} x^{2} u_{y y}(x, y, t), \\
(x, y, t) \in\left(\mathbb{R}^{+}\right)^{3}, 0<\alpha \leq 1,
\end{array}
$$

with the initial conditions

$$
u(x, y, 0)=y^{2} .
$$

Assume that $u(x, y, t)$ is an analytical function on $t>0$ and the initial approximation solution has the following form:

$$
u^{\text {initial }}(x, y, t)=y^{2} \text {. }
$$

Then the $k$ th truncated series and $k$ th residual function will be

$$
\begin{aligned}
u^{k}(x, y, t) & =u^{\text {initial }}(x, y, t)+\sum_{m=1}^{k} C_{m}(x, y) t^{m \alpha} \\
& =y^{2}+C_{1}(x, y) t^{\alpha}+\cdots+C_{k}(x, y) t^{k \alpha},
\end{aligned}
$$




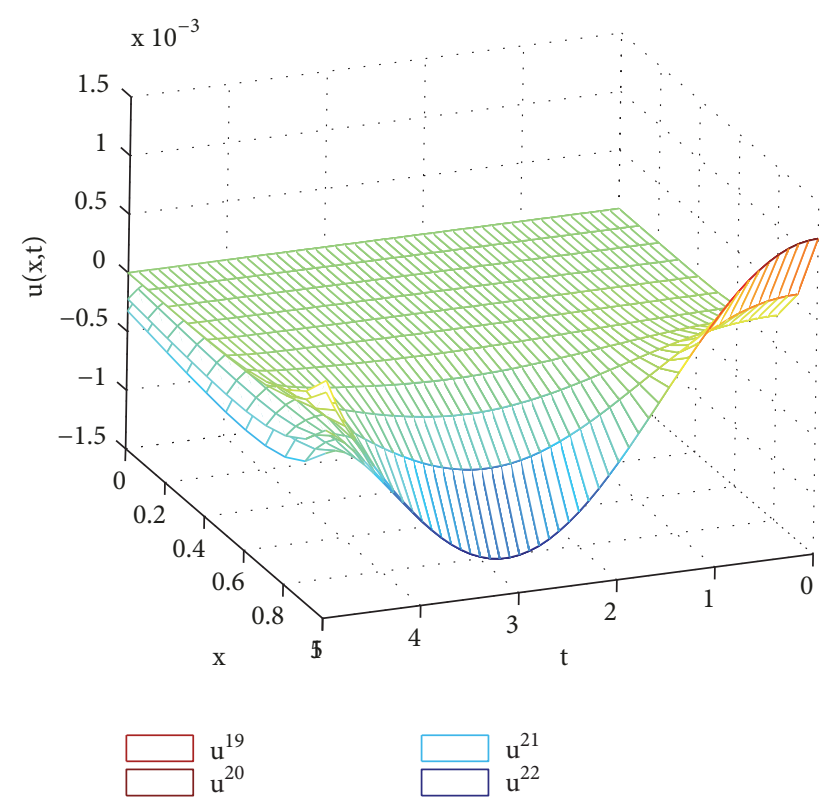

Figure 4: The approximate solutions $u^{19}, u^{20}, u^{21}$, and $u^{22}$ of (36).

and

$$
\begin{aligned}
\operatorname{Res}^{k}(x, y, t)= & D_{t}^{\alpha} u^{k}(x, y, t)-\frac{1}{2} y^{2} \frac{\partial^{2} u^{k}(x, y, t)}{\partial x^{2}} \\
& -\frac{1}{2} x^{2} \frac{\partial^{2} u^{k}(x, y, t)}{\partial y^{2}}
\end{aligned}
$$

respectively. By (18), we have

$$
D_{t}^{(k-1) \alpha} \operatorname{Res}^{k}(x, y, 0)=0,
$$

$$
0<\alpha<1, k=1,2,3, \cdots .
$$

When $k=1$ in (54), we obtain

$$
C_{1}(x, y)=\frac{x^{2}}{\Gamma(\alpha+1)}
$$

Thus, the 1st truncated approximate solution of (49)-(50) is

$$
u^{1}(x, y, t)=y^{2}+\frac{x^{2}}{\Gamma(\alpha+1)} t^{\alpha} .
$$

Let $\mathrm{k}=2$ in (54); it yields that

$$
C_{2}(x, y)=\frac{y^{2}}{\Gamma(2 \alpha+1)} .
$$

Therefore, the 2nd truncated approximate solution of (49) (50) is

$$
u^{2}(x, y, t)=y^{2}+\frac{x^{2}}{\Gamma(\alpha+1)} t^{\alpha}+\frac{y^{2}}{\Gamma(2 \alpha+1)} t^{2 \alpha} .
$$

In the similar way, taking $k=3,4,5,6$ in (54), we can obtain that

$$
\begin{aligned}
& C_{3}(x, y)=\frac{x^{2}}{\Gamma(3 \alpha+1)}, \\
& C_{4}(x, y)=\frac{y^{2}}{\Gamma(4 \alpha+1)}, \\
& C_{5}(x, y)=\frac{x^{2}}{\Gamma(5 \alpha+1)},
\end{aligned}
$$

and

$$
C_{6}(x, y)=\frac{y^{2}}{\Gamma(6 \alpha+1)}
$$

Thus 6th truncated approximate solution of (49)-(50) can be obtained

$$
\begin{aligned}
u^{6}(x, y, t)= & y^{2}+\frac{x^{2}}{\Gamma(\alpha+1)} t^{\alpha}+\frac{y^{2}}{\Gamma(2 \alpha+1)} t^{2 \alpha} \\
& +\frac{x^{2}}{\Gamma(3 \alpha+1)} t^{3 \alpha}+\frac{y^{2}}{\Gamma(4 \alpha+1)} t^{4 \alpha} \\
& +\frac{x^{2}}{\Gamma(5 \alpha+1)} t^{5 \alpha}+\frac{y^{2}}{\Gamma(6 \alpha+1)} t^{6 \alpha}
\end{aligned}
$$

Following the same step, we have the exact analytical solutions of (49)-(50):

$$
\begin{aligned}
& u(x, y, t)=y^{2}\left(1+\frac{1}{\Gamma(2 \alpha+1)} t^{2 \alpha}+\frac{1}{\Gamma(4 \alpha+1)} t^{4 \alpha}\right. \\
& \left.+\frac{1}{\Gamma(6 \alpha+1)} t^{6 \alpha}+\cdots\right)+x^{2}\left(\frac{1}{\Gamma(\alpha+1)} t^{\alpha}\right. \\
& \left.+\frac{1}{\Gamma(3 \alpha+1)} t^{3 \alpha}+\frac{1}{\Gamma(5 \alpha+1)} t^{5 \alpha}+\cdots\right)=y^{2} \\
& \cdot \cosh \left(t^{\alpha}, \alpha\right)+x^{2} \sinh \left(t^{\alpha}, \alpha\right) .
\end{aligned}
$$

Particularly, if $\alpha=1$, we obtain the following form:

$$
u(x, y, t)=y^{2} \frac{e^{t}+e^{-t}}{2}+x^{2} \frac{e^{t}-e^{-t}}{2}
$$

which is the solution of the integer order heat equation with variable coefficients.

Some numerical simulation are presented next. In Figures 5 and $6, u^{9}, u^{10}, u^{11}$, and $u^{12}$ represent the 9th-, 10th-, 11th, and 12th-order GRPS solution of (49) at time $t=3$ with $\alpha=1 / 2$ and $\alpha=1$, respectively. In Figures 7 and $8, u^{9}$, $u^{10}, u^{11}$, and $u^{12}$ represent the 9th-, 10th-, 11th-, and 12thorder GRPS solution of (49) at time $t=5$ with $\alpha=1 / 2$ and $\alpha=1$, respectively. It shows that the speed of convergence of truncated approximate solution of (49) with $\alpha=1$ is better than the one with $\alpha=1 / 2$. 


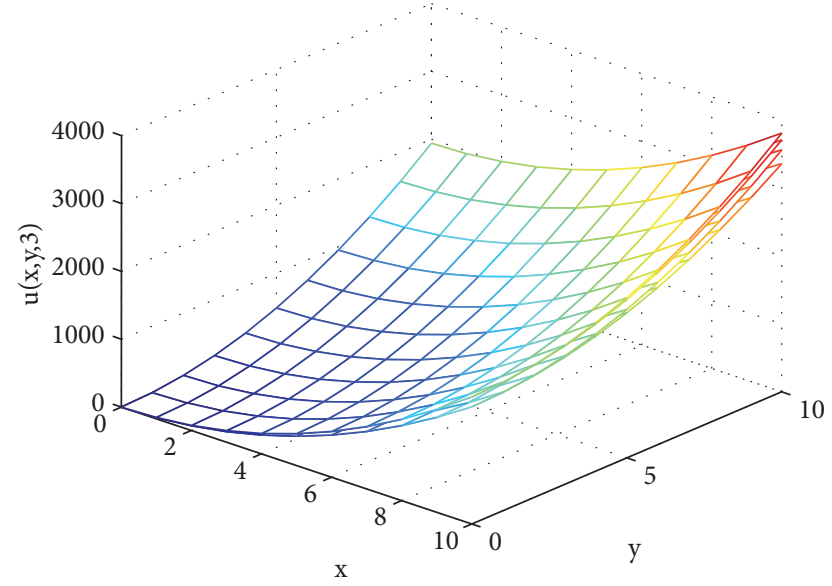

FIGURE 5: The approximate solutions $u^{9}, u^{10}, u^{11}$, and $u^{12}$ of (49) at $t=3$ with $\alpha=1 / 2$.

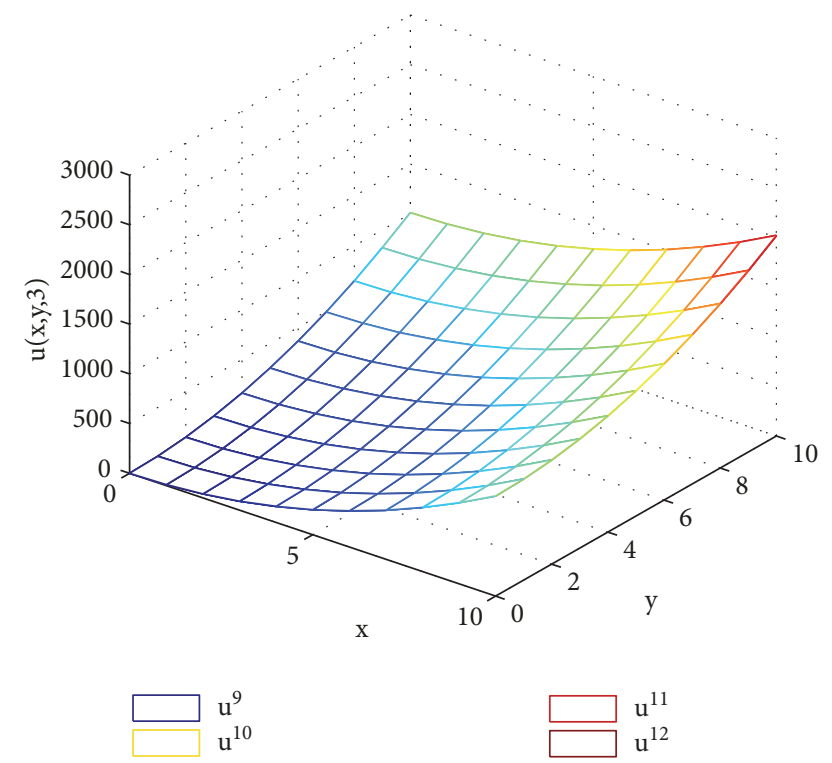

FIGURE 6: The approximate solutions $u^{9}, u^{10}, u^{11}$, and $u^{12}$ of (49) at $t=3$ with $\alpha=1$.

4.4. Fractional Wave Equation in $\mathbb{R}^{3}$. Consider the threedimensional wave equation with variable coefficients

$$
\begin{aligned}
& D_{t}^{2 \alpha} u(x, y, z, t)=\frac{1}{2}\left(x^{2} u_{x x}(x, y, z, t)\right. \\
& \left.\quad+y^{2} u_{y y}(x, y, z, t)+z^{2} u_{z z}(x, y, z, t)\right)+x^{2}+y^{2} \\
& \quad+z^{2}, \quad(x, y, z) \in\left(\mathbb{R}^{+}\right)^{3}, t>0,0<\alpha \leq 1,
\end{aligned}
$$

with the initial conditions

$$
\begin{aligned}
u(x, y, z, 0) & =0, \\
D_{t}^{\alpha} u(x, y, z, 0) & =x^{2}+y^{2}-z^{2} .
\end{aligned}
$$

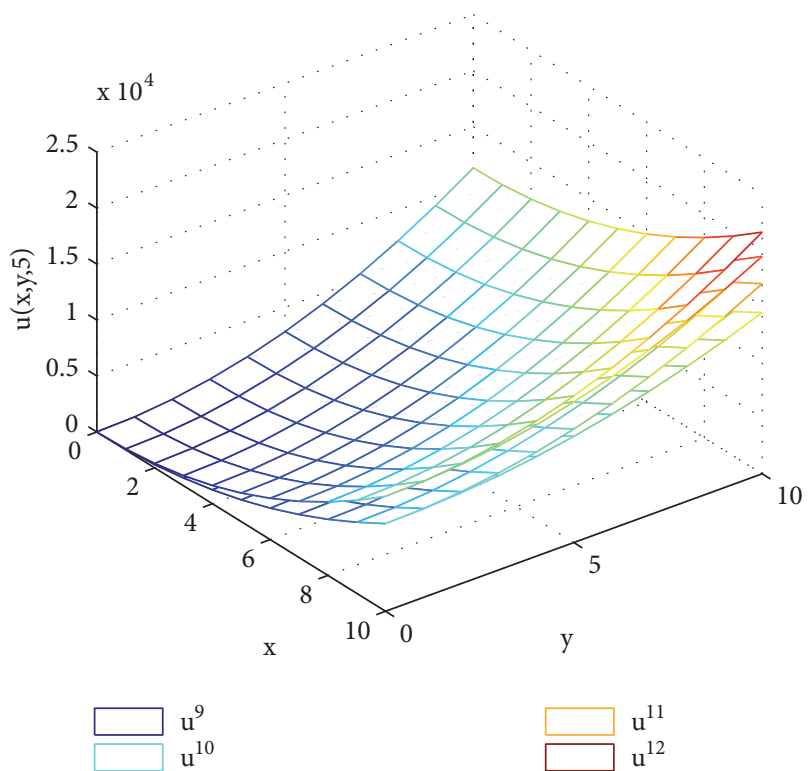

FIgURE 7: The approximate solutions $u^{9}, u^{10}, u^{11}$, and $u^{12}$ of (49) at $t=5$ with $\alpha=1 / 2$.
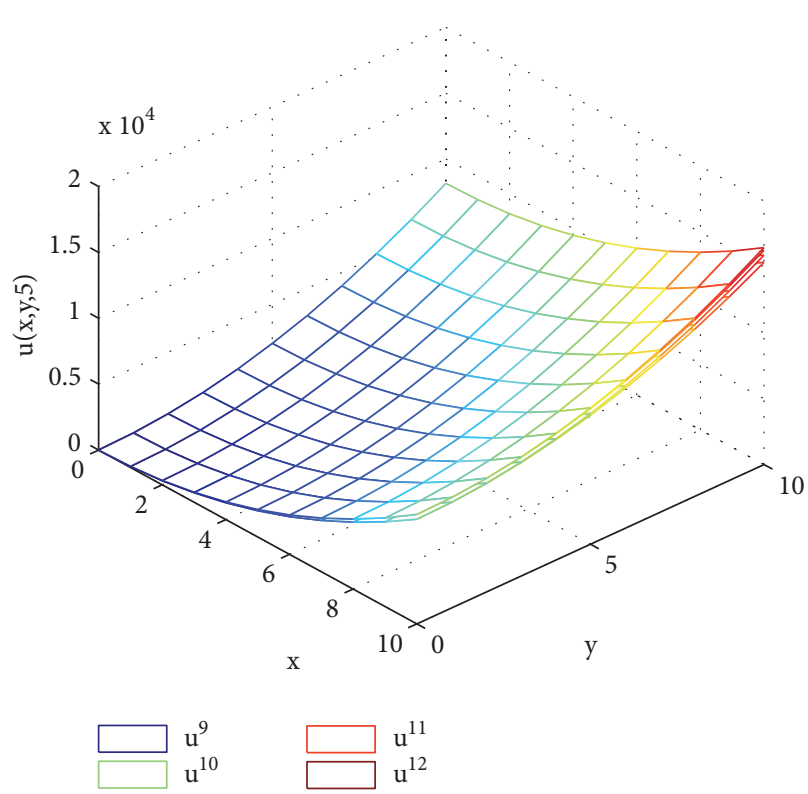

FIGURE 8: The approximate solutions $u^{9}, u^{10}, u^{11}$, and $u^{12}$ of (49) at $t=5$ with $\alpha=1$.

First, we construct the initial approximation solution:

$$
u^{\text {initial }}(x, y, z, t)=\frac{x^{2}+y^{2}-z^{2}}{\Gamma(\alpha+1)} t^{\alpha} .
$$

Secondly, construct the $k$ th truncated series and $k$ th residual function of (64)-(65) as follows:

$$
\begin{aligned}
& u^{k}(x, y, z, t)=u^{\text {initial }}(x, y, z, t) \\
& +\sum_{m=2}^{k} C_{m}(x, y, z) t^{m \alpha},
\end{aligned}
$$




$$
\begin{aligned}
\operatorname{Res}^{k} & (x, y, z, t)=D_{t}^{2 \alpha} u^{k}(x, y, z, t)-\left(x^{2}+y^{2}+z^{2}\right) \\
& -\frac{1}{2}\left(x^{2} u_{x x}^{k}(x, y, z, t)+y^{2} u_{y y}^{k}(x, y, z, t)\right. \\
& \left.+z^{2} u_{z z}^{k}(x, y, z, t)\right) .
\end{aligned}
$$

By (18), we have

$$
\begin{aligned}
D_{t}^{(k-2) \alpha} \operatorname{Res}^{k}(x, y, z, 0)= & 0, \\
& 0<\alpha<1, k=2,3,4, \cdots .
\end{aligned}
$$

Taking $k=2$ in (68), it yields

$$
C_{2}(x, y, z)=\frac{x^{2}+y^{2}+z^{2}}{\Gamma(2 \alpha+1)}
$$

Then the 2nd truncated approximate solution will be

$$
\begin{aligned}
u^{2}(x, y, z, t)= & \frac{1}{\Gamma(\alpha+1)}\left(x^{2}+y^{2}-z^{2}\right) t^{\alpha} \\
& +\frac{1}{\Gamma(2 \alpha+1)}\left(x^{2}+y^{2}+z^{2}\right) t^{2 \alpha} .
\end{aligned}
$$

In a similar way, taking $k=3,4,5,6$ in (68), we have

$$
\begin{aligned}
& C_{3}(x, y, z)=\frac{1}{\Gamma(3 \alpha+1)}\left(x^{2}+y^{2}-z^{2}\right), \\
& C_{4}(x, y, z)=\frac{1}{\Gamma(4 \alpha+1)}\left(x^{2}+y^{2}+z^{2}\right), \\
& C_{5}(x, y, z)=\frac{1}{\Gamma(5 \alpha+1)}\left(x^{2}+y^{2}-z^{2}\right), \\
& C_{6}(x, y, z)=\frac{1}{\Gamma(6 \alpha+1)}\left(x^{2}+y^{2}+z^{2}\right) .
\end{aligned}
$$

Then the 6th-order truncated approximate solution of (64)(65) can be obtained as follows:

$$
\begin{aligned}
u^{6}(x, y, z, t)= & \frac{1}{\Gamma(\alpha+1)}\left(x^{2}+y^{2}-z^{2}\right) t^{\alpha} \\
& +\frac{1}{\Gamma(2 \alpha+1)}\left(x^{2}+y^{2}+z^{2}\right) t^{2 \alpha} \\
& +\frac{1}{\Gamma(3 \alpha+1)}\left(x^{2}+y^{2}-z^{2}\right) t^{3 \alpha} \\
& +\frac{1}{\Gamma(4 \alpha+1)}\left(x^{2}+y^{2}+z^{2}\right) t^{4 \alpha} \\
& +\frac{1}{\Gamma(5 \alpha+1)}\left(x^{2}+y^{2}-z^{2}\right) t^{5 \alpha} \\
& +\frac{1}{\Gamma(6 \alpha+1)}\left(x^{2}+y^{2}+z^{2}\right) t^{6 \alpha}
\end{aligned}
$$

Finally, the exact analytical solution of (64)-(65) can be obtained:

$$
\begin{aligned}
& u(x, y, t)=\left(x^{2}+y^{2}-z^{2}\right)\left(\frac{1}{\Gamma(\alpha+1)} t^{\alpha}\right. \\
& \left.+\frac{1}{\Gamma(3 \alpha+1)} t^{3 \alpha}+\frac{1}{\Gamma(5 \alpha+1)} t^{5 \alpha}+\cdots\right)+\left(x^{2}+y^{2}\right. \\
& \left.+z^{2}\right)\left(\frac{1}{\Gamma(2 \alpha+1)} t^{2 \alpha}+\frac{1}{\Gamma(4 \alpha+1)} t^{4 \alpha}\right. \\
& \left.+\frac{1}{\Gamma(6 \alpha+1)} t^{6 \alpha}+\cdots\right)=\left(x^{2}+y^{2}-z^{2}\right) \\
& \quad \cdot \sinh \left(t^{\alpha}, \alpha\right)+\left(x^{2}+y^{2}+z^{2}\right)\left[\cosh \left(t^{\alpha}, \alpha\right)-1\right]
\end{aligned}
$$

\section{Conclusions}

This paper concerns the analytical series solutions of the differential equations with variable coefficients (integer order or fractional order). By a general residual power residual series method, we construct the analytical approximate solutions and the analytical exact solutions of the differential equations with variable coefficients, for example, nonhomogeneous parabolic equations, fractional heat equations in $2 \mathrm{D}$, and fractional wave equations in 3D. It shows that GRPS is a direct, simple, and efficient method which could be widely applied to many other PDEs with variable coefficients.

\section{Data Availability}

The Matlab data used to support the findings of this study are included within the supplementary information file.

\section{Conflicts of Interest}

The authors declare that they have no conflicts of interest regarding the publication of this paper.

\section{Acknowledgments}

This work is supported in part by NSFC Grant [11401089, 11671071] and Jilin Province's Education Department [JJKH20170535KJ, JJKH20180006KJ], JLSTDP 20160520094JH, and FRFCU2412017FZ005.

\section{Supplementary Materials}

The Matlab codes of examples 1, 2, and 3 are presented in Supplementary Material. The Matlab code of GRPS method is presented in section "Supplementary Material", which can help readers follow our simulation. (Supplementary Materials)

\section{References}

[1] V. Barbu and N. H. Pavel, "Periodic solutions to nonlinear one dimensional wave equation with $x$-dependent coefficients," Transactions of the American Mathematical Society, vol. 349, no. 5, pp. 2035-2048, 1997. 
[2] P. Gérard, "Nonlinear Schrödinger equations in inhomogeneous media: wellposedness and illposedness of the Cauchy problem," in International CONgress of Mathematicians, pp. 157182, 2006.

[3] S. Ji and Y. Li, "Periodic solutions to one-dimensional wave equation with $x$-dependent coefficients," Journal of Differential Equations, vol. 229, no. 2, pp. 466-493, 2006.

[4] K. Diethelm, The Analysis of Fractional Differential Equations. An Application-Oriented Exposition Using Differential Operators of Caputo Type, vol. 2004 of Lecture Notes in Mathematics, Springer, Berlin, Germany, 2010.

[5] J. T. Machado, V. Kiryakova, and F. Mainardi, "Recent history of fractional calculus," Communications in Nonlinear Science and Numerical Simulation, vol. 16, no. 3, pp. 1140-1153, 2011.

[6] D. Baleanu, G.-C. Wu, and S.-D. Zeng, "Chaos analysis and asymptotic stability of generalized Caputo fractional differential equations," Chaos, Solitons \& Fractals, vol. 102, pp. 99-105, 2017.

[7] V. E. Tarasov, Fractional Dynamics: Application of Fractional Calculus to Dynamics of Particles, Fields and Media, Springer, 2010.

[8] V. V. Uchaikin, "Fractional derivatives for physicists and engineers. Volume I," in Nonlinear Physical Science, Higher Education Press, Berlin, Germany, 2013.

[9] G. Adomian, "A review of the decomposition method in applied mathematics," Journal of Mathematical Analysis and Applications, vol. 135, no. 2, pp. 501-544, 1988.

[10] G. Adomian, Solving Frontier Problems of Physics: The Decomposition Method, Kluwer Academic, Dordrecht, The Netherlands, 1994.

[11] J. He, S. K. Elagan, and Z. B. Li, "Geometrical explanation of the fractional complex transform and derivative chain rule for fractional calculus," Physics Letters A, vol. 376, no. 4, pp. 257-259, 2012.

[12] S. A. Khuri, "A new approach to Bratu's problem," Applied Mathematics and Computation, vol. 147, no. 1, pp. 131-136, 2004.

[13] O. Abu Arqub, "Series solution of fuzzy differential equations under strongly generalized differentiability," Journal of Advanced Research in Applied Mathematics, vol. 5, no. 1, pp. 3152, 2013.

[14] O. Abu Arqub, Z. Abo-Hammour, R. Al-Badarneh, and S. Momani, "A reliable analytical method for solving higher-order initial value problems," Discrete Dynamics in Nature and Society, vol. 2013, Article ID 673829, 12 pages, 2013.

[15] O. Abu Arqub, "Application of residual power series method for the solution of time-fractional Schrödinger equations in onedimensional space," Fundamenta Informaticae, 2018.

[16] O. A. Arqub, A. El-Ajou, and S. Momani, "Constructing and predicting solitary pattern solutions for nonlinear time-fractional dispersive partial differential equations," Journal of Computational Physics, vol. 293, pp. 385-399, 2015.

[17] A. El-Ajou, O. Abu Arqub, and S. Momani, "Approximate analytical solution of the nonlinear fractional KdV-Burgers equation: a new iterative algorithm," Journal of Computational Physics, vol. 293, pp. 81-95, 2015.

[18] A. El-Ajou, O. Abu Arqub, S. Momani, D. Baleanu, and A. Alsaedi, "A novel expansion iterative method for solving linear partial differential equations of fractional order," Applied Mathematics and Computation, vol. 257, pp. 119-133, 2015.

[19] F. Xu, Y. Gao, X. Yang, and H. Zhang, "Construction of fractional power series solutions to fractional boussinesq equations using residual power series method," Mathematical Problems in Engineering, vol. 2016, 15 pages, 2016.
[20] L. Su, W. Wang, and Q. Xu, "Finite difference methods for fractional dispersion equations," Applied Mathematics and Computation, vol. 216, no. 11, pp. 3329-3334, 2010.

[21] C. Tadjeran, M. M. Meerschaert, and H. Scheffler, "A secondorder accurate numerical approximation for the fractional diffusion equation," Journal of Computational Physics, vol. 213, no. 1, pp. 205-213, 2006.

[22] Z. Odibat and S. Momani, "A generalized differential transform method for linear partial differential equations of fractional order," Applied Mathematics Letters, vol. 21, no. 2, pp. 194-199, 2008.

[23] S. Z. Rida, A. M. El-Sayed, and A. A. Arafa, "On the solutions of time-fractional reaction-diffusion equations," Communications in Nonlinear Science and Numerical Simulation, vol. 15, no. 12, pp. 3847-3854, 2010.

[24] Y. Chen, Y. Wu, Y. Cui, Z. Wang, and D. Jin, "Wavelet method for a class of fractional convection-diffusion equation with variable coefficients," Journal of Computational Science, vol. 1, no. 3, pp. 146-149, 2010.

[25] S. S. Ray, "Analytical solution for the space fractional diffusion equation by two-step Adomian decomposition method," Communications in Nonlinear Science and Numerical Simulation, vol. 14, no. 4, pp. 1295-1306, 2009.

[26] M. Dehghan, S. A. Yousefi, and A. Lotfi, “The use of He's variational iteration method for solving the telegraph and fractional telegraph equations," International Journal for Numerical Methods in Biomedical Engineering, vol. 27, no. 2, pp. 219-231, 2011.

[27] M. Inc, "The approximate and exact solutions of the spaceand time-fractional Burgers equations with initial conditions by variational iteration method," Journal of Mathematical Analysis and Applications, vol. 345, no. 1, pp. 476-484, 2008.

[28] M. Dehghan, J. Manafian, and A. Saadatmandi, "Solving nonlinear fractional partial differential equations using the homotopy analysis method," Numerical Methods for Partial Differential Equations, vol. 26, no. 2, pp. 448-479, 2010.

[29] S. Momani and Z. Odibat, "Comparison between the homotopy perturbation method and the variational iteration method for linear fractional partial differential equations," Computers of Mathematics with Applications, vol. 54, no. 7-8, pp. 910-919, 2007.

[30] S. K. Vanani and A. Aminataei, "Tau approximate solution of fractional partial differential equations," Computers \& Mathematics with Applications, vol. 62, no. 3, pp. 1075-1083, 2011.

[31] A. Saadatmandi and M. Dehghan, "A tau approach for solution of the space fractional diffusion equation," Computers \& Mathematics with Applications, vol. 62, no. 3, pp. 1135-1142, 2011.

[32] A. A. Kilbas, H. M. Srivastava, and J. J. Trujillo, Theory and Applications of Fractional Differential Equations, New York, NY, USA, Elsevier, 2006.

[33] K. S. Miller and B. Ross, An Introduction to the Fractional Calculus and Fractional Differential Equations, A Wiley-Interscience Publication, John Wiley \& Sons, New York, NY, USA, 1993.

[34] O. A. Arqub, A. El-Ajou, Z. A. Zhour, and S. Momani, "Multiple solutions of nonlinear boundary value problems of fractional order: a new analytic iterative technique," Entropy, vol. 16, no. 1, pp. 471-493, 2014.

[35] A.-M. Wazwaz, "Analytic treatment for variable coefficient fourth-order parabolic partial differential equations," Applied Mathematics and Computation, vol. 123, no. 2, pp. 219-227, 2001. 


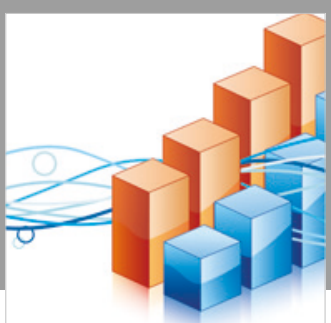

Advances in

Operations Research

\section{-n-m}
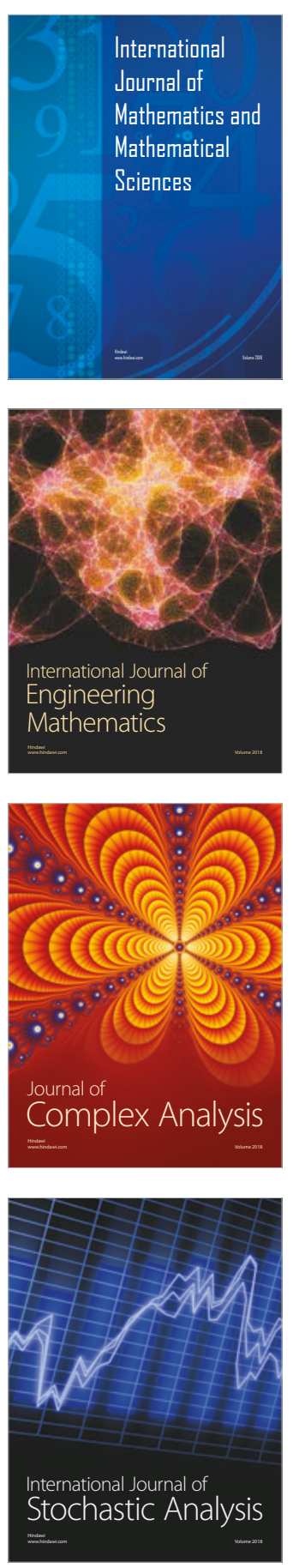
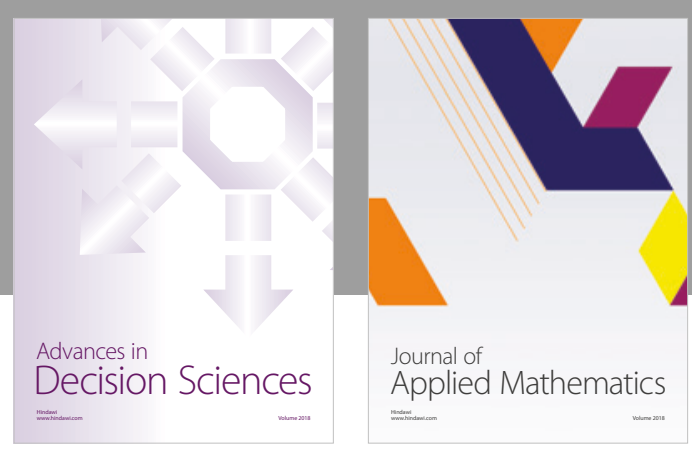

Journal of

Applied Mathematics
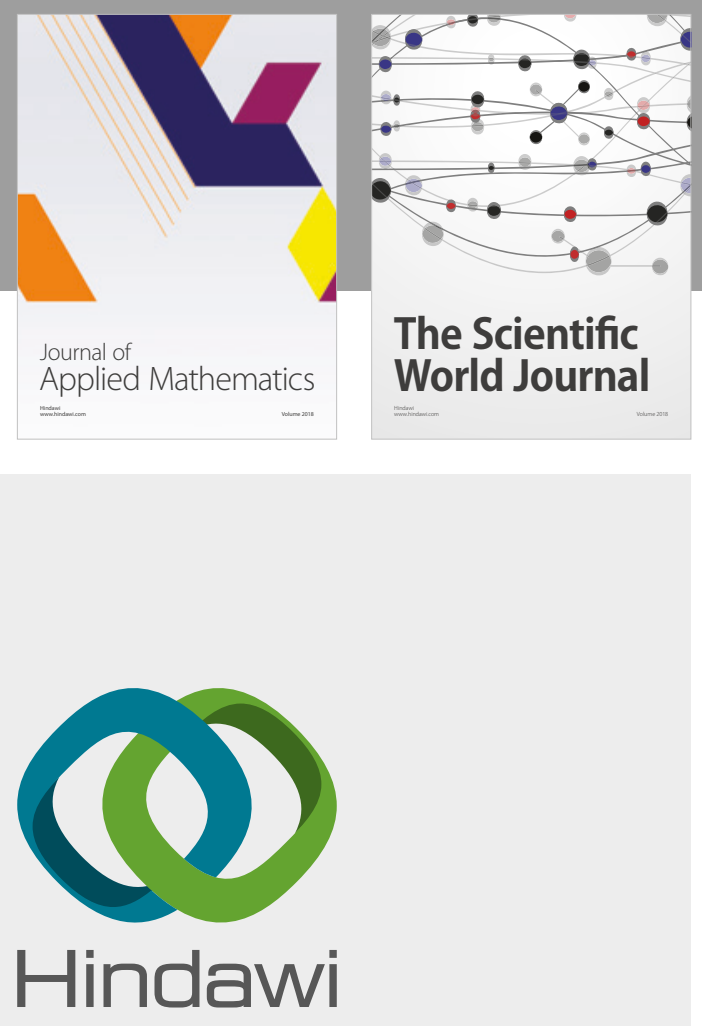

Submit your manuscripts at

www.hindawi.com

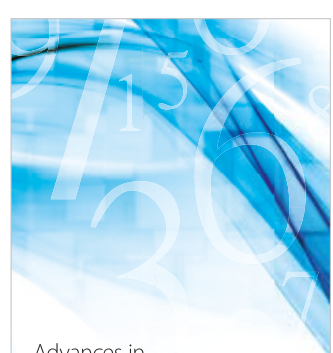

Advances in
Numerical Analysis
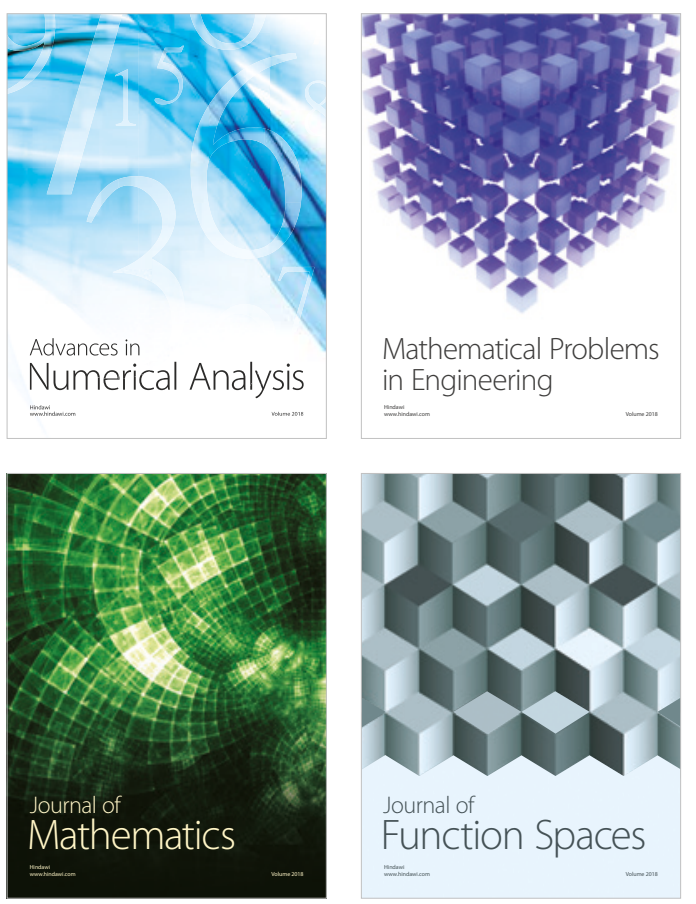

Mathematical Problems in Engineering

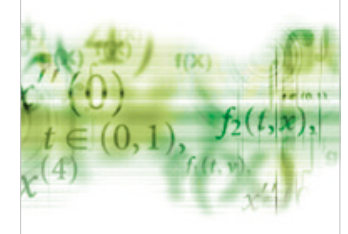

International Journal of

Differential Equations

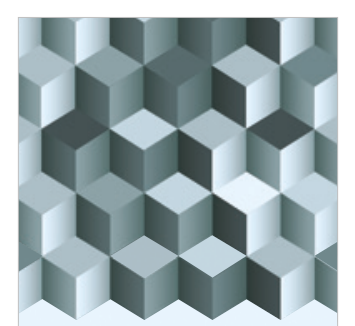

Journal of

Function Spaces

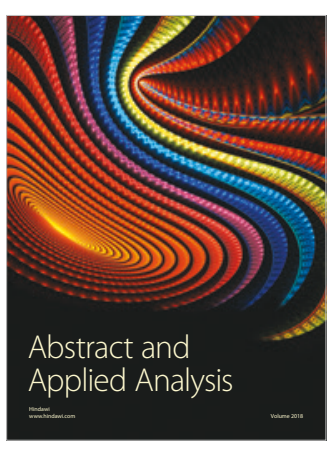

The Scientific

World Journal

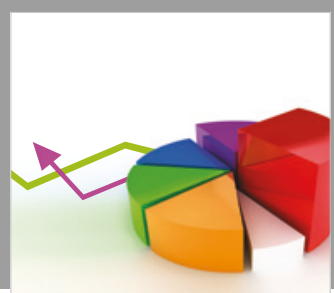

Journal of

Probability and Statistics
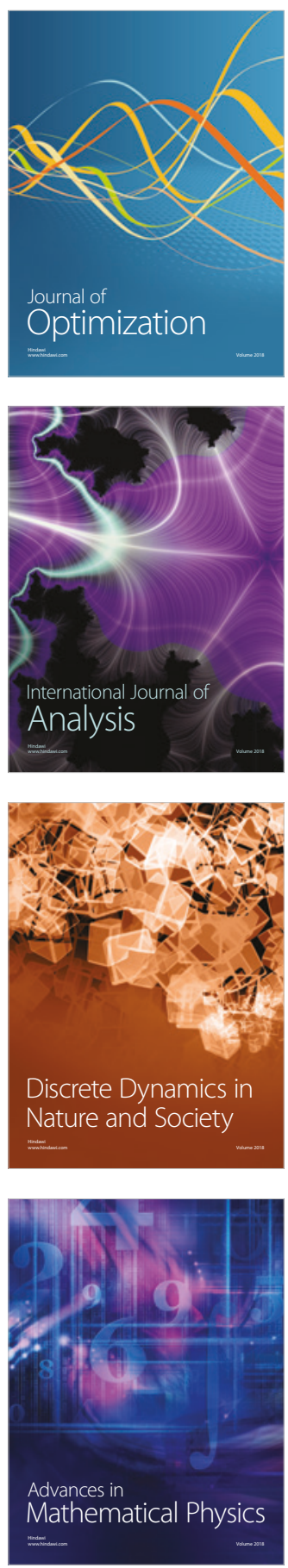